\title{
The Worst Case Finite Optimal Value in Interval Linear Programming
}

\author{
Milan Hladík ${ }^{1, *}$ \\ ${ }^{1}$ Department of Applied Mathematics, Faculty of Mathematics and Physics, Charles University, \\ Malostranské nám. 25, 11800, Prague, Czech Republic \\ E-mail: 〈hladik@kam.mff.cuni.cz〉
}

\begin{abstract}
We consider a linear programming problem, in which possibly all coefficients are subject to uncertainty in the form of deterministic intervals. The problem of computing the worst case optimal value has already been thoroughly investigated in the past. Notice that it might happen that the value can be infinite due to infeasibility of some instances. This is a serious drawback if we know a priori that all instances should be feasible. Therefore we focus on the feasible instances only and study the problem of computing the worst case finite optimal value. We present a characterization for the general case and investigate special cases, too. We show that the problem is easy to solve provided interval uncertainty affects the objective function only, but the problem becomes intractable in case of intervals in the righthand side of the constraints. We also propose a finite reduction based on inspecting candidate bases. We show that processing a given basis is still an NP-hard problem even with non-interval constraint matrix, however, the problem becomes tractable as long as uncertain coefficients are situated either in the objective function or in the right-hand side only.
\end{abstract}

Key words: linear programming, interval analysis, sensitivity analysis, interval linear programming, NP-completeness

Received: September 28, 2018; accepted: November 14, 2018; available online: December 13, 2018

DOI: $10.17535 /$ crorr.2018.0019

\section{Introduction}

Consider a linear programming (LP) problem

$$
f(A, b, c)=\min c^{T} x \text { subject to } x \in M(A, b),
$$

where $M(A, b)$ is the feasible set with constraint matrix $A \in \mathbb{R}^{m \times n}$ and the right-hand side vector $b \in \mathbb{R}^{m}$. We use the convention $\min \emptyset=\infty$ and $\max \emptyset=-\infty$. Basically, one of the following canonical forms

$$
\begin{aligned}
& f(A, b, c)=\min c^{T} x \text { subject to } A x=b, x \geq 0, \\
& f(A, b, c)=\min c^{T} x \text { subject to } A x \leq b, \\
& f(A, b, c)=\min c^{T} x \text { subject to } A x \leq b, x \geq 0
\end{aligned}
$$

is usually considered. As was repeatedly observed, in the interval setting, these forms are not equivalent to each other in general $[10,12,17]$, so they have to be analyzed separately. We can consider a general form involving all the canonical forms together [13], but from the sake of exposition, it is better to consider the canonical forms separately.

${ }^{*}$ Corresponding author. 
Interval data. An interval matrix is defined as the set

$$
\boldsymbol{A}=\left\{A \in \mathbb{R}^{m \times n} ; \underline{A} \leq A \leq \bar{A}\right\},
$$

where $\underline{A}, \bar{A} \in \mathbb{R}^{m \times n}, \underline{A} \leq \bar{A}$ are given matrices. We will use also the notion of the midpoint and radius matrix defined respectively as

$$
A_{c}:=\frac{1}{2}(\underline{A}+\bar{A}), \quad A_{\Delta}:=\frac{1}{2}(\bar{A}-\underline{A}) .
$$

The set of all $m \times n$ interval matrices is denoted by $\mathbb{I}^{m \times n}$. Similar notation is used for interval vectors, considered as one column interval matrices, and interval numbers. For interval arithmetic see, e.g., the textbooks [20, 22].

Interval linear programming. Let $\boldsymbol{A} \in \mathbb{I} \mathbb{R}^{m \times n}, \boldsymbol{b} \in \mathbb{I}^{m}$ and $\boldsymbol{c} \in \mathbb{R}^{n}$ be given. By an interval linear programming problem we mean a family of LP problems (1) with $A \in \boldsymbol{A}, b \in \boldsymbol{b}$ and $c \in \boldsymbol{c}$. A particular LP problem from this family is called a realization.

In the recent years, the optimal value range problem was intensively studied. The problem consists of determining the best case and worst case optimal values defined as

$$
\begin{aligned}
\underline{f} & :=\min f(A, b, c) \text { subject to } A \in \boldsymbol{A}, b \in \boldsymbol{b}, c \in \boldsymbol{c}, \\
\bar{f} & :=\max f(A, b, c) \text { subject to } A \in \boldsymbol{A}, b \in \boldsymbol{b}, c \in \boldsymbol{c} .
\end{aligned}
$$

The interval $\boldsymbol{f}=[f, \bar{f}]$ then gives us the range of optimal values of the interval LP problem; each realization (1) has the optimal value in $\boldsymbol{f}$. If we define the image of optimal values

$$
f(\boldsymbol{A}, \boldsymbol{b}, \boldsymbol{c}):=\{f(A, b, c) \mid A \in \boldsymbol{A}, b \in \boldsymbol{b}, c \in \boldsymbol{c}\},
$$

then the optimal value range alternatively reads

$$
\begin{aligned}
\underline{f} & :=\min f(\boldsymbol{A}, \boldsymbol{b}, \boldsymbol{c}), \\
\bar{f} & :=\max f(\boldsymbol{A}, \boldsymbol{b}, \boldsymbol{c}) .
\end{aligned}
$$

References $[6,12]$ present a survey on this topic. Methods and formulae for determining $\underline{f}$ and $\bar{f}$ were discussed in $[5,11,21,24]$. Some of the values are easily computable, but some are NP-hard, depending of the particular form $(\mathrm{A})-(\mathrm{C})$ of the LP problem. The hard cases are $\bar{f}$ for type (A) and $f$ for type (B); NP-hardness was proved in [6, 7, 26, 28]. Hladík [15] proposes approximation method for the intractable cases. Garajová et al. [10] study what is the effect of transformations of the constraints on the optimal value range, among others.

Besides the optimal value range problem also the effects on the optimal solution set were investigated. See $[2,16,19]$ for some of the recent results and the types of solutions considered.

Problem formulation. The worst case optimal value $\bar{f}$ can be infinite (i.e., $\bar{f}=\infty$ ) due to infeasibility of some realization. However, in many situations, we know a priori or can assure that all instances are feasible; a typical example is the transportation problem [4]. Therefore, we focus on feasible realizations only and define the worst case finite optimal value as

$$
\bar{f}_{\text {fin }}:=\max f(A, b, c) \text { subject to } A \in \boldsymbol{A}, b \in \boldsymbol{b}, c \in \boldsymbol{c}, f(A, b, c)<\infty .
$$

Example 1. Consider the interval LP problem

$$
\min x \text { subject to } x \leq[-1,1], x \geq 0 .
$$

Choosing a negative value from the interval $[-1,1]$, we obtain an infeasible LP problem. Choosing a nonnegative value, the resulting optimal value is zero. Therefore $f(\boldsymbol{A}, \boldsymbol{b}, \boldsymbol{c})=\{0, \infty\}$ and $\boldsymbol{f}=[\underline{f}, \bar{f}]=[0, \infty]$, but $\bar{f}_{\text {fin }}=0$. 
We will assume that there is at least one infeasible realization, that is, $f(A, b, c)=\infty$ for some $A \in \boldsymbol{A}, b \in \boldsymbol{b}$ and $c \in \boldsymbol{c}$; methods for checking this property are discussed in [6, 13], among others. Otherwise, if every realization is feasible, then $\bar{f}_{f i n}=\bar{f}$, and we can use standard techniques for computing $\bar{f}$.

\section{General results}

As the following example shows, even the value of $\bar{f}_{\text {fin }}$ can be infinite. We will show later in Proposition 5 that this happen only if there are intervals in the constraint matrix.

Example 2. Consider the interval LP problem

$$
\min -x_{1} \text { subject to }[0,1] x_{2}=-1, x_{1}-x_{2}=0, x_{1}, x_{2} \leq 0 .
$$

By direct inspection, we observe that $f(\boldsymbol{A}, \boldsymbol{b}, \boldsymbol{c})=[1, \infty]$ and $\boldsymbol{f}=[1, \infty]$. We have $\bar{f}=\infty$ because the LP problem is infeasible when choosing the zero from the interval $[0,1]$. However, we have also $\bar{f}_{f i n}=\infty$ since the optimal value $f(A, b, c) \rightarrow \infty$ as the selection from $[0,1]$ tends to zero.

Denote by

$$
g(A, b, c)=\max b^{T} y \text { subject to } y \in N\left(A^{T}, c\right)
$$

the dual problem to (1). For the canonical forms (A)-(C), the dual problems respectively read

$$
\begin{aligned}
& g(A, b, c)=\max b^{T} y \text { subject to } A^{T} y \leq c, \\
& g(A, b, c)=\max b^{T} y \text { subject to } A^{T} y=c, y \leq 0, \\
& g(A, b, c)=\max b^{T} y \text { subject to } A^{T} y \leq c, y \leq 0 .
\end{aligned}
$$

By duality in linear programming, we can replace the inner optimization problem in the definition of $\bar{f}_{f i n}$ by its dual problem with no additional assumptions. This is a bit surprising since duality in real or interval liner programming usually needs some kind of (strong) feasibility; see Novotná et al. [23].

Proposition 1. We have

$$
\bar{f}_{\text {fin }}=\max g(A, b, c) \text { subject to } A \in \boldsymbol{A}, b \in \boldsymbol{b}, c \in \boldsymbol{c}, g(A, b, c)<\infty .
$$

Proof. By strong duality in linear programming, both primal and dual problems have the same optimal value as long as at least one of them is feasible. If the primal problem is infeasible for every realization of interval data, then the dual problem is for every realization either infeasible or unbounded. In any case, both sides of (3) are equal to $-\infty$. Thus we will assume that the feasible set $M(A, b)$ is nonempty for at least one realization. The assumption ensures feasibility of at lest one realization, so we can replace the primal problem by the dual one. Notice that feasibility of all realizations is not necessary to assume since primarily infeasible instances are idle for both primal and dual problems.

The advantage of the formula (3) is that the "max min" optimization problem is reduced to "max max" problem

$$
\bar{f}_{f i n}=\max b^{T} y \text { subject to } y \in N\left(A^{T}, c\right), M(A, b) \neq \emptyset, A \in \boldsymbol{A}, b \in \boldsymbol{b}, c \in \boldsymbol{c},
$$

which can be hopefully more easy to deal with. 


\section{Special cases with $A$ real}

In this section, we focus on certain sub-classes of the main problem. In particular, we consider the case with real constraint matrix, i.e., $A_{\Delta}=0$. This case is not much on restriction on generality since the matrix $A$ characterizes the structure of the model and often is fixed. This is particularly true in transportations problems or flows in networks $[1,27]$. In contrast, costs $c$ in the objective function and capacities corresponding to the right-hand side vectors $b$ are typically affected various kinds of uncertainties.

As we already mentioned, transformations between the LP forms (A)-(C) is not equivalent in general. Nevertheless, in some cases, it is possible. Garajová et al. [10] showed that provided $A$ is real, finite optimal values (and therefore also $\bar{f}_{f i n}$ ) is not changed under the following transformations:

- transform an interval LP problem of type (A)

$$
\min \boldsymbol{c}^{T} x \text { subject to } A x=\boldsymbol{b}, x \geq 0
$$

to form (C) splitting equations to double inequalities

$$
\min \boldsymbol{c}^{T} x \text { subject to } A x \leq \boldsymbol{b}, A x \geq \boldsymbol{b}, x \geq 0,
$$

- transform an interval LP problem of type (B)

$$
\min \boldsymbol{c}^{T} x \text { subject to } A x \leq \boldsymbol{b}
$$

to form (C) by imposing nonnegativity of variables

$$
\min \boldsymbol{c}^{T} x^{+}-\boldsymbol{c}^{T} x^{-} \text {subject to } A x^{+}-A x^{-} \leq \boldsymbol{b}, x^{+}, x^{-} \geq 0 .
$$

In Garajová et al. [10], it was also observed that the first transformation may change finite optimal values in the case with interval $\boldsymbol{A}$. Below, we show by an example that this is also true for the second transformation.

Example 3. Consider the interval LP problem of type (B)

$$
\min -x \text { subject to }[0,1] x \leq-1,-[1,2] x \leq 5 .
$$

It is easy to see that $\boldsymbol{f}=[1,5] \cup\{\infty\}$ and $\bar{f}_{\text {fin }}=5$. Imposing nonnegativity of variables leads to the interval LP problem

$$
\min -x^{+}+x^{-} \text {subject to }[0,1] x^{+}-[0,1] x^{-} \leq-1,-[1,2] x^{+}+[1,2] x^{-} \leq 5 .
$$

Now, the set of optimal values expands significantly. For instance, the realization

$$
\min -x^{+}+x^{-} \text {subject to } 0.1 x^{+}-0.1 x^{-} \leq-1,-2 x^{+}+1 x^{-} \leq 5
$$

has the optimal value of 10 . By direct inspection, we can see that $\boldsymbol{f}=\{-\infty\} \cup[1, \infty]$. That is, the worst case finite optimal value grows to $\bar{f}_{\text {fin }}=\infty$.

\subsection{Interval objective function}

If interval data are situated in the objective vector only, computation of $\bar{f}_{f i n}$ is easy just by solving one LP problem.

Proposition 2. If $A$ and $b$ are real, then computation of $\bar{f}_{f i n}$ is a polynomial problem. 
Proof. Under the assumptions, the problem (4) takes the form of an LP problem in variables $x, y, c$. Moreover, the variable $c$ can be easily eliminated. For types (A) and (C) in particular, the resulting LP problem draw, respectively

$$
\begin{aligned}
& \bar{f}_{f i n}=\max b^{T} y \text { subject to } A x=b, x \geq 0, A^{T} y \leq \bar{c}, \\
& \bar{f}_{f i n}=\max b^{T} y \text { subject to } A x \leq b, x \geq 0, A^{T} y \leq \bar{c}, y \leq 0 .
\end{aligned}
$$

For type (B) we have

$$
\bar{f}_{\text {fin }}=\max b^{T} y \text { subject to } A x \leq b, \underline{c} \leq A^{T} y \leq \bar{c}, y \leq 0 .
$$

Corollary 1. Suppose that $A$ and $b$ are real and $M(A, b) \neq \emptyset$. For interval LP problems of types $(A)$ and $(C)$ the value of $\bar{f}_{\text {fin }}$ is attained at $c:=\bar{c}$.

Proof. Due to $M(A, b) \neq \emptyset$, problems (5) and (6) take respectively the form of

$$
\begin{array}{ll}
\bar{f}_{f i n}=\max b^{T} y & \text { subject to } A^{T} y \leq \bar{c}, \\
\bar{f}_{f i n}=\max b^{T} y & \text { subject to } A^{T} y \leq \bar{c}, y \leq 0 .
\end{array}
$$

Again by $M(A, b) \neq \emptyset$, we can replace the LP problems by their duals

$$
\begin{array}{ll}
\bar{f}_{\text {fin }}=\min \bar{c}^{T} x & \text { subject to } A x=b, x \geq 0, \\
\bar{f}_{\text {fin }}=\min \bar{c}^{T} x & \text { subject to } A x \leq b, x \geq 0 .
\end{array}
$$

The LP problems on the right-hand sides yield $\bar{f}_{f i n}$ for the corresponding LP forms.

Notice that for LP problems of type (B), this property is not true. In general, $\bar{f}_{\text {fin }}$ is not attained for extremal values of $\boldsymbol{c}$, which is illustrated by the following example.

Example 4. Consider the interval LP problem of type (B)

$$
\min -x_{1}+c_{2} x_{2} \text { subject to } x_{1}+x_{2} \leq 2,-x_{1}+x_{2} \leq 0,
$$

where $c_{2} \in \boldsymbol{c}_{2}=-[0.5,2]$. It is not hard to see that $\bar{f}_{\text {fin }}=\bar{f}=-2$, and it is attained for the value of $c_{2}:=-1$ at the point $x=(1,1)^{T}$. For smaller $c_{2}$, the optimal value is $-1+c_{2}<-2$. For larger $c_{2}$, the optimal value is $-\infty$ since the problem is unbounded.

\subsection{Interval right-hand side}

In contrast to the previous case, if interval data are situated in the right-hand side vector only (i.e., $A_{\Delta}=0$ and $c_{\Delta}=0$ ), computation of $\bar{f}_{f i n}$ is intractable.

Proposition 3. If $A$ and $c$ are real, then checking $\bar{f}_{\text {fin }}>0$ is NP-hard for type (A).

Proof. By [9], checking whether there is at least one feasible realization of the interval system

$$
A^{T} y \leq 0, \boldsymbol{b}^{T} y>0
$$

is an NP-hard problem. Hence it is NP-hard to check $\bar{f}>0$ (not yet speaking about $\bar{f}_{\text {fin }}$ ) for the interval LP problem

$\max \boldsymbol{b}^{T} y$ subject to $A^{T} y \leq 0$. 
Due to positive homogeneity of the constraints, we can rewrite the problem as

$$
\max \boldsymbol{b}^{T} y \text { subject to } A^{T} y \leq 0, y \leq e,-y \leq e,
$$

where $e=(1, \ldots, 1)^{T}$. For this interval problem, checking $\bar{f}_{\text {fin }}>0$ is NP-hard.

The interval problem (7) follows the form (3); the condition $g(A, b, c)<\infty$ needn't be considered since the problem is feasible and finite for each realization. Thus we can view this problem as the dual of an interval LP problem of type (A), which has a fixed objective function vector and a fixed constraint matrix.

Corollary 2. If $A$ and $c$ are real, then checking $\bar{f}_{f i n}>0$ is NP-hard for type (B) and for type $(C)$.

Proof. By Proposition 3, checking $\bar{f}_{f i n}>0$ is NP-hard for an interval LP problem

$$
\min c^{T} x \text { subject to } A x=\boldsymbol{b}, x \geq 0 .
$$

According to the discussion at the beginning of Section 3 , the value of $\bar{f}_{f i n}$ is not changed under the transformation of equations to double inequalities

$$
\min c^{T} x \text { subject to } A x \leq \boldsymbol{b}, A x \geq \boldsymbol{b}, x \geq 0 .
$$

This is, however, a type $(\mathrm{C})$ problem, which must therefore be NP-hard.

Type (B) problems are also NP-hard since every problem in the form of $(\mathrm{C})$ is essentially in the form of $(B)$.

Despite intractability, computation of $\bar{f}_{\text {fin }}$ need not be always so hard. If $A$ is real, then (4) takes the form of a bilinear programming problem, that is, the constraints are linear and the objective function is bilinear (with respect to variables $y, b, c$ ). Even though it is NP-hard, some instances may be faster solvable.

Example 5. Consider an interval LP problem in the form

$$
\min c^{T} x \text { subject to } A x \geq \boldsymbol{b}
$$

with $\underline{b}>0$. Then (4) reads

$$
\bar{f}_{f i n}=\max b^{T} y \text { subject to } A x \geq b, A^{T} y=c, y \geq 0, b \in \boldsymbol{b} .
$$

Since the variables are nonnegative, it has the special form of a geometric program, and hence it is efficiently solvable [3].

\section{Basis approach}

If the LP problem (1) has a finite optimal value, then it possesses an optimal solution corresponding to an optimal basis. For concreteness, consider type (A) problem. A basis $B$ is optimal if and only if the following two conditions are satisfied

$$
\begin{aligned}
A_{B}^{-1} b & \geq 0, \\
c_{N}^{T}-c_{B}^{T} A_{B}^{-1} A_{N} & \geq 0^{T} .
\end{aligned}
$$

The optimal value then is $f(A, b, c)=c_{B}^{T} A_{B}^{-1} b$.

Given a basis $B$ and an interval LP problem, we will now address the question what is the highest optimal value achievable at this basis. This can be formulated as an optimization problem

$$
\max c_{B}^{T} A_{B}^{-1} b \text { subject to (8), } A \in \boldsymbol{A}, b \in \boldsymbol{b}, c \in \boldsymbol{c} .
$$


Real constraint matrix. Suppose from now on that $A$ is real. Then the optimization problem (9) reads

$$
\max c_{B}^{T} A_{B}^{-1} b \text { subject to }(8), b \in \boldsymbol{b}, c \in \boldsymbol{c} .
$$

Its constrains are linear in variables $b, c$. Therefore, checking its feasibility is an easy task. In accordance with [12], we say that a basis $B$ is weakly optimal if it admits at least one finite optimal value, that is, $B$ is optimal for some realization. From the above reasoning, we have

Proposition 4. Checking whether a basis B is weakly optimal is a polynomial problem.

The feasible set of (10) is bounded, so the optimal value is bounded, too. Since there are finitely many basis, the worst case finite optimal value must be finite. Hence we just derived

Proposition 5. If $A$ is real, then $\bar{f}_{\text {fin }}<\infty$.

If $c$ is real, then (9) takes the form of an LP problem

$$
\max c_{B}^{T} A_{B}^{-1} b \text { subject to }(8), b \in \boldsymbol{b},
$$

and so it is polynomially solvable. Similarly in the case when $b$ is real.

Proposition 6. If $A, b$ are real or $A, c$ are real, then solving (9) is polynomial.

Solving problem (9) with $A$ real and $\boldsymbol{b}, \boldsymbol{c}$ interval values is, however, still intractable.

Proposition 7. If $A$ is real, then solving (9) is NP-hard.

Proof. By Witsenhausen [29], it is NP-hard to find the maximum value of a bilinear form $u^{T} M v$ on interval domain $u, v \in[0,1]^{n}$, where $M$ is symmetric nonsingular. We will reduce this problem to our problem. We put $\boldsymbol{b}:=[0,1]^{n}$ and $A_{B}:=I_{n}$, where $I_{n}$ is the identity matrix. Next, we substitute $c_{B}:=M u$. The condition

$$
c_{B}=M u, u \in[0,1]^{n}
$$

is equivalent to

$$
0 \leq M^{-1} c_{B} \leq 1
$$

so we can formulate it as (8b) for $A_{N}=\left(M^{-1},-M^{-1}\right)$ and $c_{N}=\left(1^{T}, 0^{T}\right)^{T}$. The condition (8a) is trivially satisfied as $A_{B}^{-1} b=b \in[0,1]^{n}$. This completes the reduction.

Real $A$ and $c$. By Proposition 3 we know that computing $\bar{f}_{\text {fin }}$ is NP-hard even when $A$ and $c$ are real, and intervals are situated in the right-hand side vector $\boldsymbol{b}$ only. The above considerations give us a finite reduction for computing $\bar{f}_{\text {fin }}$ : For each basis $B$, check if it is weakly optimal and determine the worst case optimal value associated with $B$ by solving the LP problem (11).

In this way, the box $\boldsymbol{b}$ splits into convex polyhedral sub-parts, which are usually called stability or critical regions in the context of sensitivity analysis and parametric programming [8]. Each region corresponds to a weakly optimal basis. In the area of interval linear programming, but in another context, stability regions were also discussed in Mráz [21].

The obvious drawback of this approach is that there are exponentially many bases. On the other hand, the number of weakly optimal bases might be reasonably small. In order to process them, consider the following graph. The nodes correspond to weakly optimal bases. There is an edge between two nodes if and only if the corresponding bases are neighbors, that is, they differ in exactly one entry (the basic index sets differ in one entry). Since the set $\boldsymbol{b}$ of the objective vectors of the dual problem (2) is convex and compact, the graph of weakly 


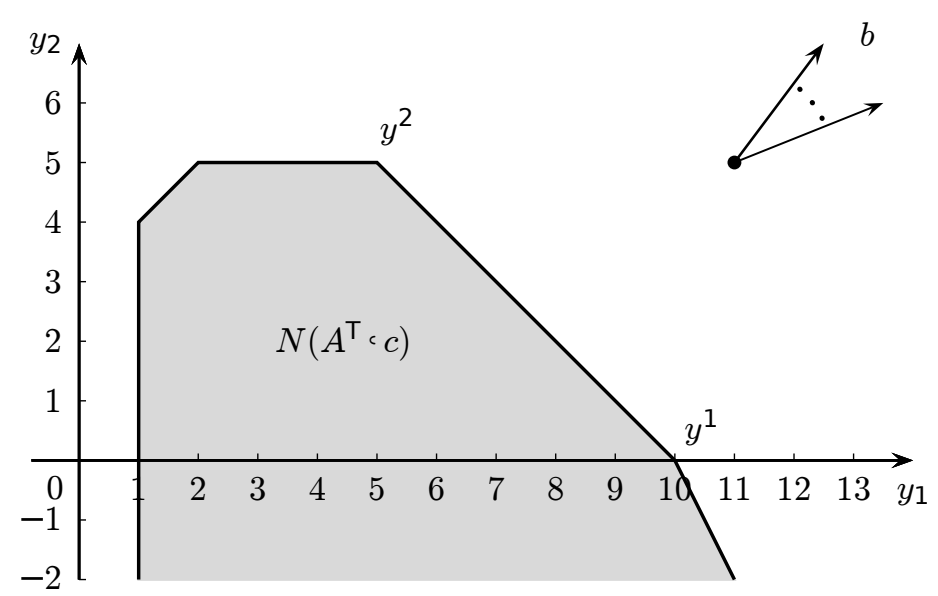

Figure 1: (Example 6) Illustration of the dual problem: for different values of the objective vector b, the optimal solution moves from $y^{1}$ to $y^{2}$ and to unbounded instances.

optimal bases is connected. Therefore, we can start with one weakly optimal basis, inspect the neighboring bases for weak optimality and process until all weakly optimal bases are found.

This method can be significantly faster than processing all possible bases. In particular, if the interval vector $\boldsymbol{b}$ is narrow, then we can expect that the number of weakly optimal basis is small, or even there is a unique one. This case of unique basis is called basis stable problem and was investigated in $[14,18,25]$. Even though it is NP-hard to check for basis stability of a basis $B$ for a general interval LP problem, there are practically efficient sufficient conditions; see [14].

Moreover, basis stability is polynomially decidable provided $A, b$ or $A, c$ are real, which is our case. Concretely, we have to verify two conditions. First, check (8b), which is easy as all data are constant. Second, compute by interval arithmetic the expression $A_{B}^{-1} \boldsymbol{b}$, and check that the lower bound is nonnegative.

Example 6. Consider the interval LP problem of type (A) with data

$$
A=\left(\begin{array}{ccccc}
1 & 2 & 0 & -1 & -1 \\
1 & 1 & 1 & 1 & 0
\end{array}\right), \quad \boldsymbol{b}=\left(\begin{array}{l}
{[3,5]} \\
{[2,4]}
\end{array}\right), \quad c=\left(\begin{array}{llllll}
10 & 20 & 5 & 3 & 1
\end{array}\right)^{T} .
$$

The dual problem is illustrated on Figure 1. There are two weakly optimal bases, $B=\{1,2\}$ and $B^{\prime}=\{1,3\}$. On the figure, they correspond to vertices $y^{1}=(10,0)^{T}$ and $y^{2}=(5,5)^{T}$.

For basis $B$, the constraint $A_{B}^{-1} b \geq 0$ from (8a) takes the form

$$
\begin{aligned}
-b_{1}+2 b_{2} & \geq 0, \\
b_{1}-b_{2} & \geq 0 .
\end{aligned}
$$

By the LP problem (11), we compute the value of the highest optimal value corresponding to this basis as 50 .

For basis $B^{\prime}$, the constraint $A_{B}^{-1} b \geq 0$ draws

$$
\begin{aligned}
b_{1} & \geq 0, \\
-b_{1}+b_{2} & \geq 0 .
\end{aligned}
$$

The LP problem (11) now gives the value of 40 for the highest optimal value associated to $B^{\prime}$.

In total, we see that the worst case optimal value is $\bar{f}_{\text {fin }}=50$ and it is attained for basis $B$. Figure 2 depicts the interval vector $\boldsymbol{b}$ and its subparts corresponding to the optimal bases $B$ and $B^{\prime}$ and to infeasible instances. 


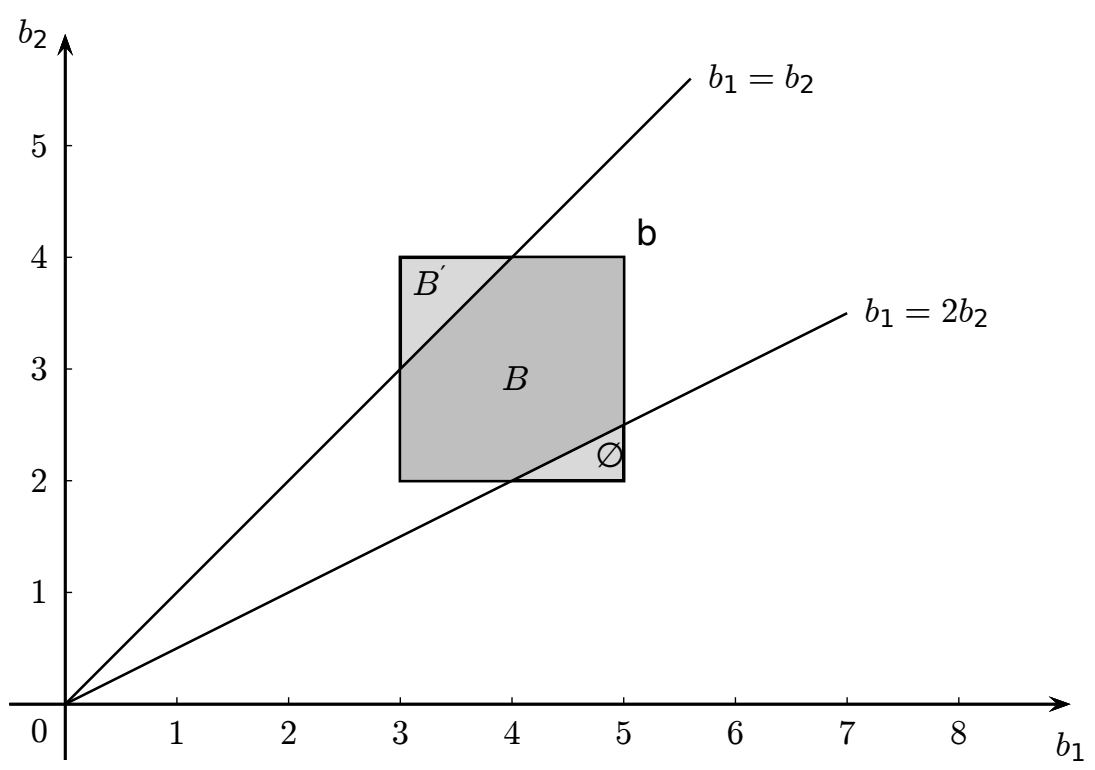

Figure 2: (Example 6) The sub-parts of interval vector $\boldsymbol{b}$ corresponding to the optimal bases $B$ and $B^{\prime}$ and to infeasible instances.

\section{Conclusion}

We investigated the problem of computing the highest possible optimal value when input data are subject to variations in given intervals and we restrict to feasible instances only. We analyzed the computational complexity issues by identifying the cases that are already polynomially solvable and those that are still NP-hard. The basis reduction proposes an approach that is not a priori exponential even for the NP-hard cases.

Several open questions arised during the work on the topic. This includes for example the problem of what is the computational complexity of this question: Is $\bar{f}_{f i n}$ attained for a given basis $B$ ?

\section{Acknowledgement}

The author was supported by the Czech Science Foundation Grant P403-18-04735S.

\section{References}

[1] Ahuja, R. K., Magnanti, T. L. and Orlin, J. B. (1993). Network Flows. Theory, Algorithms, and Applications. Englewood Cliffs, NJ: Prentice Hall.

[2] Ashayerinasab, H. A., Nehi, H. M. and Allahdadi, M. (2018). Solving the interval linear programming problem: A new algorithm for a general case. Expert Systems with Applications, 93, Suppl. C, 39-49.

[3] Boyd, S. and Vandenberghe, L. (2004). Convex Optimization. Cambridge University Press.

[4] Cerulli, R., D'Ambrosio, C. and Gentili, M. (2017). Best and worst values of the optimal cost of the interval transportation problem. In Sforza, A., and Sterle, C. (Eds.), Optimization and Decision Science: Methodologies and Applications, volume 217 of Springer Proceedings in Mathematics \& Statistics, (pp. 367-374). Cham: Springer.

[5] Chinneck, J. W. and Ramadan, K. (2000). Linear programming with interval coefficients. Journal of the Operational Research Society, 51(2), 209-220. 
[6] Fiedler, M., Nedoma, J., Ramík, J., Rohn, J. and Zimmermann, K. (2006). Linear Optimization Problems with Inexact Data. New York: Springer.

[7] Gabrel, V. and Murat, C. (2010). Robustness and duality in linear programming. Journal of the Operational Research Society, 61(8), 1288-1296.

[8] Gal, T. and Greenberg, H. J. (Eds.) (1997). Advances in Sensitivity Analysis and Parametric Programming. Boston: Kluwer Academic Publishers.

[9] Garajová, E., Hladík, M. and Rada, M. (2017). On the properties of interval linear programs with a fixed coefficient matrix. In Sforza, A., and Sterle, C. (Eds.), Optimization and Decision Science: Methodologies and Applications, volume 217 of Springer Proceedings in Mathematics \& Statistics, (pp. 393-401). Cham: Springer.

[10] Garajová, E., Hladík, M. and Rada, M. (2018). Interval linear programming under transformations: Optimal solutions and optimal value range. Central European Journal of Operations Research. In press, doi: 10.1007/s10100-018-0580-5

[11] Hladík, M. (2009). Optimal value range in interval linear programming. Fuzzy Optimization and Decision Making, 8(3), 283-294.

[12] Hladík, M. (2012). Interval linear programming: A survey. In Mann, Z. A. (Ed.), Linear Programming - New Frontiers in Theory and Applications, chapter 2, (pp. 85-120). New York: Nova Science Publishers.

[13] Hladík, M. (2013). Weak and strong solvability of interval linear systems of equations and inequalities. Linear Algebra and its Applications, 438(11), 4156-4165.

[14] Hladík, M. (2014). How to determine basis stability in interval linear programming. Optimization Letters, 8(1), 375-389.

[15] Hladík, M. (2014). On approximation of the best case optimal value in interval linear programming. Optimization Letters, 8(7), 1985-1997.

[16] Hladík, M. (2017). On strong optimality of interval linear programming. Optimization Letters, $11(7), 1459-1468$.

[17] Hladík, M. (2017). Transformations of interval linear systems of equations and inequalities. Linear and Multilinear Algebra, 65(2), 211-223.

[18] Koníčková, J. (2001). Sufficient condition of basis stability of an interval linear programming problem. ZAMM, Z. Angew. Mathematics and Mechanics of Solids, 81, Suppl. 3, 677-678.

[19] Li, W., Liu, X. and Li, H. (2015). Generalized solutions to interval linear programmes and related necessary and sufficient optimality conditions. Optimization Methods and Software, 30(3), 516530 .

[20] Moore, R. E., Kearfott, R. B., and Cloud, M. J. (2009). Introduction to Interval Analysis. Philadelphia, PA: SIAM.

[21] Mráz, F. (1998). Calculating the exact bounds of optimal values in LP with interval coefficients. Annals of Operations Research, 81, 51-62.

[22] Neumaier, A. (1990). Interval Methods for Systems of Equations. Cambridge: Cambridge University Press.

[23] Novotná, J., Hladík, M. and Masařík, T. (2017). Duality gap in interval linear programming. In Zadnik Stirn et al., L. (Ed.), Proceedings of the 14th International Symposium on Operational Research SOR'17, Bled, Slovenia, September 27-29, 2017, (pp. 501-506). Ljubljana, Slovenia: Slovenian Society Informatika.

[24] Rohn, J. (1984). Interval linear systems. Freiburger Intervall-Berichte 84/7, Albert-LudwigsUniversität, Freiburg.

[25] Rohn, J. (1993). Stability of the optimal basis of a linear program under uncertainty. Operations Research Letters, 13(1), 9-12.

[26] Rohn, J. (1997). Complexity of some linear problems with interval data. Reliable Computing, $3(3), 315-323$.

[27] Schrijver, A. (2004). Combinatorial Optimization. Polyhedra and efficiency, volume 24 of Algorithms and Combinatorics. Berlin: Springer.

[28] Serafini, P. (2005). Linear programming with variable matrix entries. Operations Research Letters, $33(2), 165-170$.

[29] Witsenhausen, H. S. (1986). A simple bilinear optimization problem. Systems \& Control Letters, $8(1), 1-4$. 